\title{
THE SOLUTION OF BOUNDARY VALUE PROBLEMS BY A DOUBLE LAPLACE TRANSFORMATION
}

\author{
J. C. JAEGER
}

1. Introduction. The Laplace transformation has most frequently been used to transform a linear partial differential equation with two independent variables and constant coefficients into an ordinary differential equation, the "subsidiary equation," from the solution of which that of the original equation is deduced. If it is applied to an equation with more than two independent variables, the subsidiary equation is also a partial differential equation and is usually solved by classical methods. The object of this note is to point out that partial differential equations in which the range of two (or more) independent variables is $(0, \infty)$ may easily be handled by simultaneous Laplace transformations in these variables. The point of view is that of previous papers ${ }^{1}$ in which the Laplace transformation method has been regarded as purely formal, and the solution as subject to verification. The method is related to that of Doetsch ${ }^{2}$ for equations of elliptic type but assumes no theoretical basis. In $\$ 3$ a simple two variable problem is discussed to illustrate the method and in $\$ \$ 4$ and 5 are given two new three variable problems of a type to which it is well adapted.

2. The general method. We consider a function $v(x, t)$ in the range $x>0, t>0$.

Let

$$
\begin{array}{ll}
u_{0}(x)=v(x, 0), & u_{1}(x)=\left[\frac{\partial v}{\partial t}\right]_{t=0}, \\
w_{0}(t)=v(0, t), & w_{1}(t)=\left[\frac{\partial v}{\partial x}\right]_{x=0} .
\end{array}
$$

The Laplace transform with respect to $t$ will be denoted by a "bar," thus

$$
\bar{f}(p)=\int_{0}^{\infty} e^{-p t} f(t) d t,
$$

and that with respect to $x$ by a capital letter, thus

${ }^{1}$ Carslaw and Jaeger, Proceedings of the Cambridge Philosophical Society, vol. 35 (1939), p. 394; Proceedings of the London Mathematical Society, in press; this Bulletin, vol. 45 (1939), p. 407.

2 Doetsch, Theorie und Anwendungen der Laplace-Transformation, Berlin, 1937, chap. 22. 


$$
G\left(p^{\prime}\right)=\int_{0}^{\infty} e^{-p^{\prime} x} g(x) d x
$$

and the double Laplace transform by both:

$$
\bar{V}\left(p^{\prime}, p\right)=\int_{0}^{\infty} \int_{0}^{\infty} e^{-p t-p^{\prime} x} v(x, t) d x d t
$$

$p$ and $p^{\prime}$ are supposed to have real parts sufficiently large to ensure convergence. This notation indicates compactly the variables occurring; if there is another independent variable $y$, it will occur throughout.

Then subject to suitable restrictions on $v$ we have

$$
\begin{aligned}
& \int_{0}^{\infty} \int_{0}^{\infty} e^{-p t-p^{\prime} x} \frac{\partial v}{\partial t} d x d t=p \bar{V}-U_{0}, \\
& \int_{0}^{\infty} \int_{0}^{\infty} e^{-p t-p^{\prime} x} \frac{\partial^{2} v}{\partial t^{2}} d x d t=p^{2} \bar{V}-p U_{0}-U_{1}, \\
& \int_{0}^{\infty} \int_{0}^{\infty} e^{-p t-p^{\prime} x} \frac{\partial v}{\partial x} d x d t=p^{\prime} \bar{V}-\bar{w}_{0}, \\
& \int_{0}^{\infty} \int_{0}^{\infty} e^{-p t-p^{\prime} x} \frac{\partial^{2} v}{\partial x^{2}} d x d t=p^{\prime 2} \bar{V}-p^{\prime} \bar{w}_{0}-\bar{w}_{1} .
\end{aligned}
$$

From a given differential equation and boundary conditions we obtain a subsidiary equation and boundary conditions by multiplying by $\exp \left[-p t-p^{\prime} x\right]$, integrating with respect to $x$ and $t$ from 0 to $\infty$, and using (1) to (4). Solving this equation we obtain $\bar{V}\left(p^{\prime}, p\right)$.

To derive $v(x, t)$ from $\bar{V}\left(p^{\prime}, p\right)$ we assume an inversion theorem which may be derived formally from Fourier's integral theorem in several variables:

$$
v(x, t)=-\frac{1}{4 \pi^{2}} \int_{\gamma-i \infty}^{\gamma+i \infty} d \lambda \int_{\gamma^{\prime}-i \infty}^{\gamma^{\prime}+i \infty} e^{\lambda t+\lambda^{\prime} x} \bar{V}\left(\lambda^{\prime}, \lambda\right) d \lambda^{\prime},
$$

where $\pi>\arg \lambda>-\pi$, and $\pi>\arg \lambda^{\prime}>-\pi$, provided that:

$$
\begin{array}{r}
\bar{V}\left(\lambda^{\prime}, \lambda\right) \text { is bounded in some half-planes } R\left(\lambda^{\prime}\right)>\alpha^{\prime}, R(\lambda)>\alpha, \\
\text { and } \gamma>\alpha, \gamma^{\prime}>\alpha^{\prime} .
\end{array}
$$

Other conditions in addition to (6) will of course be necessary for the truth of the inversion theorem (5), but neither these nor the assumptions involved in (1) to (4) need be discussed since the whole process of determining $v(x, t)$ as a double contour integral of type (5) 
is regarded as purely formal. It has then to be verified that this solution does satisfy the differential equation and boundary conditions. This is done in the case of the equation of conduction of heat by transforming the paths of integration in (5) to paths beginning in the third and ending in the second quadrant, and the verification is then performed on these new integrals. The whole process follows the lines of the one-variable case ${ }^{3}$ and will not be given here. The contour integrals of type (5) are evaluated by a deformation of the contour precisely as in the one-variable case, this will be done without comment, the method and justification having been given in the papers referred to.

3. Heat conduction in the region $x>0$; the initial temperature zero; the end $x=0$ kept at unit temperature for $t>0$. We have to solve

$$
\frac{\partial^{2} v}{\partial x^{2}}-\frac{1}{\kappa} \frac{\partial v}{\partial t}=0, \quad t>0, x>0
$$

with $^{4}$

$$
u_{0}(x)=0, \quad x>0,
$$

By (1) and (4) the subsidiary equation is

$$
\left(p^{\prime 2}-\frac{p}{\kappa}\right) \bar{V}=\frac{p^{\prime}}{p}+\bar{w}_{1}
$$

and so

$$
\bar{V}\left(p^{\prime}, p\right)=\frac{p^{\prime}+p \bar{w}_{1}(p)}{p\left(p^{\prime 2}-p / \kappa\right)} .
$$

For $\bar{V}\left(\lambda^{\prime}, \lambda\right)$ to satisfy (6) we must have $\bar{w}_{1}(\lambda)=-(\lambda \kappa)^{-1 / 2}$ and thus

$$
\bar{V}\left(\lambda^{\prime}, \lambda\right)=\frac{1}{\lambda\left[\lambda^{\prime}+(\lambda / \kappa)^{1 / 2}\right]} .
$$

Hence by the inversion theorem (5)

\footnotetext{
${ }^{3}$ Carslaw and Jaeger, loc. cit.

${ }^{4}$ Boundary conditions stated shortly as in (8) and (9) are to be understood as:

$$
\begin{array}{ll}
\lim _{t \rightarrow 0} v(x, t)=0, & \text { for fixed } x>0, \\
\lim _{x \rightarrow 0} v(x, t)=1, & \text { for fixed } t>0 .
\end{array}
$$

${ }^{5}$ From this result the flux of heat at the origin may be obtained directly.
} 


$$
\begin{aligned}
v(x, t) & =-\frac{1}{4 \pi^{2}} \int_{\gamma-i \infty}^{\gamma+i \infty} d \lambda \int_{\gamma^{\prime}-i \infty}^{\gamma^{\prime}+i \infty} \frac{e^{\lambda t+\lambda^{\prime} x} d \lambda^{\prime}}{\lambda\left[\lambda^{\prime}+(\lambda / \kappa)^{1 / 2}\right]} \\
& =\frac{1}{2 \pi i} \int_{\gamma-i \infty}^{\gamma+i \infty} e^{\lambda t-x(\lambda / \kappa)^{1 / 2}} \frac{d \lambda}{\lambda} \\
& =1-\frac{2}{\pi^{1 / 2}} \int_{0}^{x / 2(\kappa t)^{1 / 2}} e^{-\xi^{2}} d \xi .
\end{aligned}
$$

4. Heat conduction in the region $x>0,0<y<b ; y=b$ maintained at unit temperature for $t>0 ; y=0$ and $x=0$ maintained at zero for $t>0$; the initial temperature zero. We have to solve

with

$$
\frac{\partial^{2} v}{\partial x^{2}}+\frac{\partial^{2} v}{\partial y^{2}}-\frac{1}{\kappa} \frac{\partial v}{\partial t}=0, \quad t>0, x>0,0<y<b,
$$

$$
\begin{aligned}
& u_{0}(y, x)=0 \text {, } \\
& x>0,0<y<b, \\
& w_{0}(y, t)=0 \text {, } \\
& t>0,0<y<b \text {, } \\
& w_{1}(y, t) \text { unknown, } \\
& v(y, x, t)=0 \text {, } \\
& \text { when } y=0, x>0, t>0 \text {, } \\
& v(y, x, t)=1 \text {, } \\
& \text { when } y=b, x>0, t>0 \text {. }
\end{aligned}
$$

The subsidiary equation is

$$
\frac{d^{2} \bar{V}}{d y^{2}}+q^{2} \bar{V}=\bar{w}_{1}(y)
$$

where $q^{2}=p^{\prime 2}-p / \kappa$. This is to be solved with $\bar{V}=1 / p p^{\prime}$ when $y=b$ and $\bar{V}=0$ when $y=0$. The solution is

$$
\begin{aligned}
\bar{V}\left(y, p^{\prime}, p\right)= & \frac{\sin q y}{p p^{\prime} \sin q b}-\frac{\sin q y}{q \sin q b} \int_{y}^{b} \sin q\left(b-y^{\prime}\right) \bar{w}_{1}\left(y^{\prime}\right) d y^{\prime} \\
& -\frac{\sin q(b-y)}{q \sin q b} \int_{0}^{y} \sin q y^{\prime} \bar{w}_{1}\left(y^{\prime}\right) d y^{\prime} .
\end{aligned}
$$

Now $\sin b\left(\lambda^{\prime 2}-\lambda / \kappa\right)^{1 / 2}$, qua function of $\lambda^{\prime}$, has zeros at $\lambda^{\prime}=$ $\pm\left(n^{2} \pi^{2} / b^{2}+\lambda / \kappa\right)^{1 / 2}, n=0,1, \cdots$, so to satisfy (6) the numerator of $\bar{V}\left(y, \lambda^{\prime}, \lambda\right)$ must vanish at $\lambda^{\prime}=+\left(n^{2} \pi^{2} / b^{2}+\lambda / \kappa\right)^{1 / 2}, n=1,2, \cdots$, that is,

$$
\begin{aligned}
\frac{(-)^{n} n \pi}{\lambda b\left(n^{2} \pi^{2} / b^{2}+\lambda / \kappa\right)^{1 / 2}}+\int_{0}^{b} \sin \frac{n \pi y^{\prime}}{b} \bar{w}_{1}\left(y^{\prime}\right) d y^{\prime} & =0, \\
n & =1,2, \ldots .
\end{aligned}
$$


The residue of $e^{\lambda^{\prime} x} \bar{V}\left(y, \lambda^{\prime}, \lambda\right)$, qua function of $\lambda^{\prime}$ at the pole $\lambda^{\prime}=-\left(n^{2} \pi^{2} / b^{2}+\lambda / \kappa\right)^{1 / 2}$ is

$$
\begin{aligned}
\frac{\exp \left[-x\left(n^{2} \pi^{2} / b^{2}+\lambda / \kappa\right)^{1 / 2}\right]}{b\left(n^{2} \pi^{2} / b^{2}+\lambda / \kappa\right)^{1 / 2}} \sin \frac{n \pi y}{b}\left\{\frac{(-)^{n} n \pi}{\lambda b\left(n^{2} \pi^{2} / b^{2}+\lambda / \kappa\right)^{1 / 2}}\right. \\
\left.-\int_{0}^{b} \sin \frac{n \pi y^{\prime}}{b} \bar{w}_{1}\left(y^{\prime}\right) d y^{\prime}\right\} .
\end{aligned}
$$

Using (13) in this gives for the result of the $\lambda^{\prime}$-integration in (5)

$$
\begin{aligned}
& \frac{\sinh y(\lambda / \kappa)^{1 / 2}}{\lambda \sinh b(\lambda / \kappa)^{1 / 2}}+\sum_{n=1}^{\infty} \frac{2 \kappa n \pi(-)^{n}}{\lambda\left(\kappa n^{2} \pi^{2}+\lambda b^{2}\right)} \\
& \cdot \sin \frac{n \pi y}{b} \exp \left[-x\left(\frac{n^{2} \pi^{2}}{b^{2}}+\frac{\lambda}{\kappa}\right)^{1 / 2}\right],
\end{aligned}
$$

where the first term comes from the pole $\lambda^{\prime}=0$.

For the $\lambda$-integration we require

$$
\begin{aligned}
& \frac{1}{2 \pi i} \int_{\gamma-i \infty}^{\gamma+i \infty} \frac{\exp \left[\lambda t-x\left(\lambda / \kappa+\alpha^{2}\right)^{1 / 2}\right] d \lambda}{\lambda\left(\lambda / \kappa+\alpha^{2}\right)} \\
& \quad=\frac{1}{\alpha^{2}} e^{-\alpha x}-\frac{1}{\alpha^{2}} e^{-\kappa \alpha^{2} t}+\frac{2}{\pi} \int_{0}^{\infty} e^{-\kappa t\left(\alpha^{2}+u^{2}\right)} \sin u x \frac{d u}{u\left(u^{2}+\alpha^{2}\right)},
\end{aligned}
$$

and

$$
\frac{1}{2 \pi i} \int_{\gamma-i \infty}^{\gamma+i \infty} \frac{e^{\lambda t}}{\lambda} \frac{\sinh y(\lambda / \kappa)^{1 / 2}}{\sinh b(\lambda / \kappa)^{1 / 2}} d \lambda=\frac{y}{b}+\frac{2}{\pi} \sum_{n=1}^{\infty} \frac{(-)^{n}}{n} e^{-\kappa n^{2} \pi^{2} t / b^{2}} \sin \frac{n \pi y}{b} .
$$

Using these results we have finally

$$
\begin{aligned}
v(y, x, t)=\frac{y}{b}+ & \frac{2}{\pi} \sum_{n=1}^{\infty} e^{-n \pi x / b} \frac{(-)^{n}}{n} \sin \frac{n \pi y}{b} \\
+ & \frac{4}{b^{2}} \sum_{n=1}^{\infty} n(-)^{n} \sin \frac{n \pi y}{b} \\
& \cdot \int_{0}^{\infty} e^{-\kappa t\left(u^{2}+n^{2} \pi^{2} / b^{2}\right)} \frac{\sin u x}{u\left(u^{2}+n^{2} \pi^{2} / b^{2}\right)} d u .
\end{aligned}
$$

Clearly problems in which the temperature of $y=b$ is a function of $x$ or $t$ may be dealt with in the same way provided the function satisfies fairly wide conditions.

5. Heat conduction in the region $x>0,0<y<b$; the initial tem- 
perature unity; the surface maintained at zero for $t>0$. We have to solve

$$
\frac{\partial^{2} v}{\partial x^{2}}+\frac{\partial^{2} v}{\partial y^{2}}-\frac{1}{\kappa} \frac{\partial v}{\partial t}=0, \quad t>0, x>0,0<y<b
$$

with

$$
\begin{array}{ll}
u_{0}(y, x)=1, & x>0,0<y<b, \\
w_{0}(y, t)=0, & t>0,0<y<b, \\
w_{1}(y, t) \text { unknown, } &
\end{array}
$$$$
v(y, x, t)=0 \text { when } y=0 \text { and } y=b \text { for } x>0 \text { and } t>0 \text {. }
$$

The subsidiary equation is

$$
\frac{d^{2} \bar{V}}{d y^{2}}+q^{2} \bar{V}=\bar{w}_{1}(y)-\frac{1}{\kappa p^{\prime}}
$$

where $q^{2}=p^{\prime 2}-p / \kappa$.

This is to be solved with $\bar{V}=0$, when $y=0$ and $y=b$. The solution is

$$
\begin{aligned}
\bar{V}\left(y, p^{\prime}, p\right)= & -\frac{\sin q b-\sin q y-\sin q(b-y)}{\kappa p^{\prime} q^{2} \sin q b} \\
& -\frac{\sin q y}{q \sin q b} \int_{y}^{b} \sin q\left(b-y^{\prime}\right) \bar{w}_{1}\left(y^{\prime}\right) d y^{\prime} \\
& -\frac{\sin q(b-y)}{q \sin q b} \int_{0}^{y} \sin q y^{\prime} \bar{w}_{1}\left(y^{\prime}\right) d y^{\prime}
\end{aligned}
$$

The zeros of $\sin b\left(\lambda^{\prime 2}-\lambda / \kappa\right)^{1 / 2}$, qua function of $\lambda^{\prime}$, are at $\lambda^{\prime}$ $= \pm\left(n^{2} \pi^{2} / b+\lambda / \kappa\right)^{1 / 2}, n=0,1,2, \cdots$, and thus, in order that (6) may be satisfied, we must have

$$
\begin{aligned}
-\frac{b\left[1-(-)^{n}\right]}{n \pi \kappa\left(n^{2} \pi^{2} / b^{2}+\lambda / \kappa\right)^{1 / 2}}+\int_{0}^{b} \sin \frac{n \pi y^{\prime}}{b} \bar{w}_{1}\left(y^{\prime}\right) d y^{\prime} & =0, \\
n & =1,2, \cdots .
\end{aligned}
$$

The residue of $e^{\lambda^{\prime} x} \bar{V}\left(y, \lambda^{\prime}, \lambda\right)$ at the pole $\lambda^{\prime}=-\left(n^{2} \pi^{2} / b^{2}+\lambda / \kappa\right)^{1 / 2}$ is

$$
\begin{aligned}
-\frac{1}{b}\left(\frac{n^{2} \pi^{2}}{b^{2}}+\frac{\lambda}{\kappa}\right)^{-1 / 2} \exp \left[-x\left(\frac{n^{2} \pi^{2}}{b^{2}}+\frac{\lambda}{\kappa}\right)\right] \sin \frac{n \pi y}{b} \\
\cdot\left\{\frac{b\left[1-(-)^{n}\right]}{\kappa n \pi\left(n^{2} \pi^{2} / b^{2}+\lambda / \kappa\right)^{1 / 2}}+\int_{0}^{b} \sin \frac{n \pi y^{\prime}}{b} \bar{w}_{1}\left(y^{\prime}\right) d y^{\prime}\right\} .
\end{aligned}
$$

Using (16) in this the result of the $\lambda^{\prime}$-integration in (5) is 


$$
\begin{aligned}
-\frac{4 b^{2}}{\pi} \sum_{r=0}^{\infty} \frac{1}{(2 r+1)\left[\kappa(2 r+1)^{2} \pi^{2}+\lambda b^{2}\right]} \\
\quad \cdot \exp \left[-x\left(\frac{(2 r+1)^{2} \pi^{2}}{b^{2}}+\frac{\lambda}{\kappa}\right)^{1 / 2}\right] \sin \frac{(2 r+1) \pi y}{b} \\
+\frac{\sinh b(\lambda / \kappa)^{1 / 2}-\sinh y(\lambda / \kappa)^{1 / 2}-\sinh (b-y)(\lambda / \kappa)^{1 / 2}}{\lambda \sinh b(\lambda / \kappa)^{1 / 2}},
\end{aligned}
$$

where the last term has been derived from the pole $\lambda^{\prime}=0$; there are no poles at $\lambda^{\prime}= \pm(\lambda / \kappa)^{1 / 2}$.

Carrying out the $\lambda$-integration in the usual way, we have finally

$$
\begin{aligned}
v(y, x, t)= & \frac{8}{\pi^{2}} \sum_{r=0}^{\infty} \frac{\sin (2 r+1) \pi y / b}{(2 r+1)} \\
& \int_{0}^{\infty} \frac{\sin u x}{u} \exp \left[-\kappa t\left(u^{2}+(2 r+1)^{2} \pi^{2} / b^{2}\right)\right] d u .
\end{aligned}
$$

The same method applies to cases in which the initial temperature $u_{0}(y, x)$ is a function of $x$ and $y$ under fairly general conditions on the function.

As an example let $u_{0}(y, x)=x$, for $x>0,0<y<b$.

The only change in $\bar{V}\left(y, p^{\prime}, p\right)$ is that the first term of (15) is replaced by

$$
\frac{-\sin b q+\sin q y+\sin q(b-y)}{\kappa p^{\prime 2} q^{2} \sin b q} .
$$

Proceeding in the same way, we find that the only pole of $\bar{V}\left(y, \lambda^{\prime}, \lambda\right)$, qua function of $\lambda^{\prime}$, is $\lambda^{\prime}=0$, where the residue is

$$
x \frac{\sinh b(\lambda / \kappa)^{1 / 2}-\sinh y(\lambda / \kappa)^{1 / 2}-\sinh (b-y)(\lambda / \kappa)^{1 / 2}}{\lambda \sinh b(\lambda / \kappa)^{1 / 2}} .
$$

Performing the $\lambda$-integration of (5), we obtain finally

$$
v(y, x, t)=\frac{4 x}{\pi} \sum_{r=0}^{\infty} \frac{1}{2 r+1} e^{-\kappa \pi^{2}(2 r+1)^{2} t / b^{2}} \sin \frac{(2 r+1) \pi y}{b} .
$$

The University of Tasmania 